\title{
$k$-MERSIONS OF MANIFOLDS ${ }^{1}$
}

BY SIDNIE DRESHER FEIT

Communicated by G. D. Mostow, September 26, 1967

Let $M^{n}$ be an $n$-dimensional $C^{\infty}$ manifold and $W^{p}$ be a $p$-dimensional $C^{\infty}$ manifold. A $C^{\infty}$ mapping $f: M^{n} \rightarrow W^{p}$ is called a $k$-mersion if its rank is greater than or equal to $k$ everywhere. The set of $k$ mersions, endowed with the $C^{1}$ topology, is denoted $R\left(M^{n}, W^{p} ; k\right)$. A $k$-regular homotopy between $k$-mersions $f$ and $g$ is a continuous mapping $F: I \rightarrow R\left(M^{n}, W^{p} ; k\right)$ such that $F(0)=f$ and $f(1)=g$.

A $k$-bundle map, $\psi: T M^{n} \rightarrow T W^{p}$ between the tangent spaces of $M^{n}$ and $W^{p}$ is a continuous fibre preserving mapping such that the restriction of $\psi$ to any fibre is a linear map of rank at least $k$. The space of $k$-bundle maps with the compact open topology is denoted $T\left(M^{n}, W^{p} ; k\right)$.

An $n$-mersion is an immersion, and an $n$-regular homotopy is usually called a regular homotopy. In 1958 and 1959, Smale [4], [5] published papers classifying immersions of spheres in Euclidean spaces. Smale proved that if $n<p$, the regular homotopy classes of immersions of $S^{n}$ in $E^{p}$ are in one to rne correspondence with the homotopy classes of sections of $S^{n}$ into the bundle associated with $T S^{n}$ whose fibre is the Stiefel manifold $V_{p, n}$ of $n$ frames in $p$-dimensional Euclidean space. Smale obtained this classification by proving a stronger result, namely, that the map $d: R\left(S^{n}, E^{p} ; n\right) \rightarrow T\left(S^{n}, E^{p} ; n\right)$ defined by $d(f)=d f$ is a weak homotopy equivalence if $n<p$. His proof was based on the diagram

$$
\begin{gathered}
R\left(S^{n}, E^{p} ; n\right) \stackrel{d}{\rightarrow} T\left(S^{n}, E^{p} ; n\right) \\
\downarrow i^{*} \quad \\
R\left(D^{n}, E^{p} ; n\right) \stackrel{d}{\rightarrow} T\left(D^{*}, E^{p} ; n\right)
\end{gathered}
$$

where $D^{n}$ is identified with a hemisphere of $S^{n}$, and $i^{*}$ and $j^{*}$ are restriction maps. The main step in the proof consists of showing that $i^{*}$

1 This work was performed in partial fulfillment of the requirements for the degree of Doctor of Philosophy in Cornell University, 1967. I wish to thank Professor R. Szczarba of Yale University, under whose direction this work was done. 
and $j^{*}$ are fiberings (i.e., have covering homotopy property). Then it is easily shown that the $d$ of the bottom row is a weak homotopy equivalence and that $d$ restricted to a fibre of $i^{*}$ is a weak homotopy equivalence. It follows immediately from the homotopy sequence of a bundle and the five lemma that the $d$ of the top row is a weak homotopy equivalence.

In 1959, Hirsch [1] extended this result to the case of immersions $R\left(M^{n}, W^{p} ; n\right)$ of a $C^{\infty}$ manifold in another, with $\operatorname{dim} M^{n}<\operatorname{dim} W^{p}$, (i.e. $n<p$ ) and $\partial W^{p}=\varnothing$. Poenaru's exposition of this result [3] was the basis of Phillips' thesis in 1965 (published as [2]) which stated that if $M^{n}$ has no compact components with empty boundary and $\partial W^{p}=\varnothing$, then $d: R\left(M^{n}, W^{p} ; p\right) \rightarrow T\left(M^{n}, W^{p} ; p\right)$ is a weak homotopy equivalence. Phillips called the maps whose rank equalled the dimension of the image space "submersions."

Poenaru's exposition also is the basis of the generalization given here.

Theorem 1. Let $M^{n}$ and $W^{p}$ be $C^{\infty}$ manifolds with $\partial W^{p}=\varnothing$. The mapping $d: R\left(M^{n}, W^{p} ; k\right) \rightarrow T\left(M^{n}, W^{p} ; k\right)$ defined by $d(f)=d f$ is a weak homotopy equivalence if either

(a) $M^{n}$ has no compact components with empty boundary, or

(b) $k<p$.

Corollary 1. If condition (a) or condition (b) of Theorem 1 is satisfied, the k-regular homotopy classes of $k$-mersions of $M^{n}$ in $W^{p}$ are in one to one correspondence with the homotopy classes of $k$-bundle maps of $T M^{n}$ in $T W^{p}$.

Denote by $M^{*}(p, n ; k)$ the set of $p \times n$ matrices of rank at least $k$.

Corollary 2. Suppose condition (a) or condition (b) is satisfied. The $k$-regular homotopy classes of $k$-mersions of $M^{n}$ in $R^{p}$ are in one to one correspondence with the homotopy classes of sections of $M^{n}$ into the bundle associated with $T M^{n}$ whose fibre is $M^{*}(p, n ; k)$.

As an application of the above, it can be shown that if $p$ $\geqq(3 / 2)(n-1)$, there exists an $n-1$ mersion of $M^{n}$ in $R^{p}$.

The proof of Theorem 1 uses a filtration of $M^{n}$

$$
D^{n}=U_{0}^{n} \subset U_{1}^{n} \subset U_{2}^{n} \cdots \subset M^{n}
$$

where $U_{i}$ is obtained from $U_{i-1}$ by adding a handle, essentially. The simple scheme of (1) is replaced by 


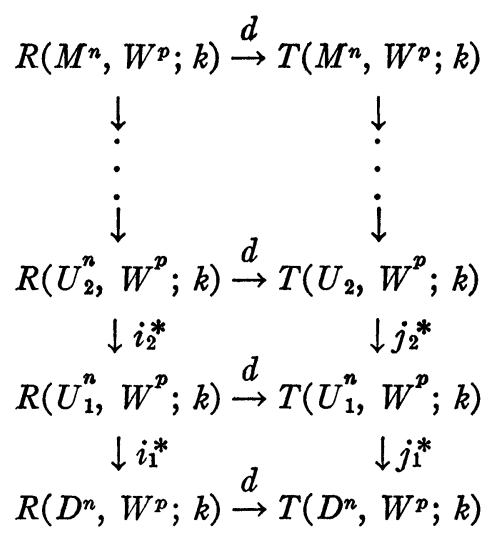

It is easy to show that the $d$ of the bottom row is a weak homotopy equivalence, and that all of the $j^{*}$ maps are fiberings. The main step in the proof is in showing that the $i^{*}$ maps are fiberings from which it also easily follows that $d$ restricted to a fibre is a weak homotopy equivalence. The rough outlines of the proof that the $i^{*}$ maps are fiberings are as follows. Let $V^{n}$ be obtained from $U^{n}$ by adding a handle of index $\lambda$. Denote $I^{m}=([0,1])^{m}$, and $I^{m-1}([0,1])^{m-1} \times\{0\}$. The map $i^{*}$ has the covering homotopy property if, given continuous maps $g: I^{m} \rightarrow R\left(U^{n}, W^{p} ; k\right)$ and $G: I^{m-1} \rightarrow R\left(V^{n}, W^{p} ; k\right)$ such that $G(q) \mid U^{n}=g(q)$ when $q \in I^{m-1}$, there is a mapping $\bar{G}: I^{m} \rightarrow R\left(V^{n}, W^{p} ; k\right)$ with

$$
\bar{G}(q)=G(q), \quad q \in I^{m-1}, \quad \bar{G}(q) \mid U^{n}=g(q), \quad q \in I^{m} .
$$

$\bar{G}(q)$ is an extension of $g(q)$ over $V$, so $\bar{G}$ can be viewed as a continuously varying set of extensions of $g$ over $V$ which agrees with $G$ on $I^{m-1}$.

If, for each $f \in R\left(U^{n}, W^{p} ; k\right)$ there is a neighborhood $\eta(f)$ for which $i^{*}{ }_{\eta(f)}=\left(i^{*} \mid i^{*-1}(\eta(f))\right)$ has the covering homotopy property, it is very easy to show that $i^{*}$ has the covering homotopy property. Thus, given $f \in R\left(U^{n}, W^{p} ; k\right)$ it suffices to find a neighborhood $\eta$ of $f$ such that if $g$ and $G$ map into $\eta$ and $i^{*-1}(\eta)$ respectively, an extension $\bar{G}$ satisfying (3) can always be constructed.

As a first step, it is not difficult to show that there is a neighborhood $\eta$ of $f$ whose elements can be factored through automorphisms of some compact $(n+p)$-dimensional manifold $C$. In fact, there is a compact neighborhood $N^{n}$ of $U^{n}$ in $V^{n}$, an embedding $s: N^{n} \rightarrow C$, a differentiable map $P: C \rightarrow W^{p}$, and a continuous mapping $\nu: \eta \rightarrow$ Aut $C$ (the automorphisms of $C$ which equal the identity near $\partial C$ ) so that $P \circ \nu(h) \circ s \mid U=h, h \in \eta$. 


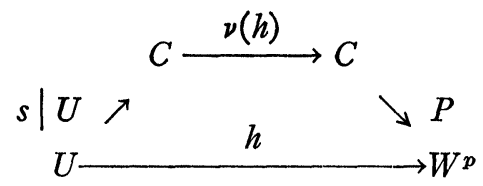

Note that $P \circ \nu(h) \circ s$ is an extension of $h$ to $N^{n}$, and if $g: I^{m} \rightarrow \eta$, then $P \circ \nu(g(q)) \circ s$ is a continuously varying set of extensions of $g$ over $N$. It turns out that $\nu$ can be modified so that $P \circ \nu(g(q)) \circ s$ $=G(q) \mid N$ if $q \in I^{m-1}$. Thus the factorization (4) enables a lift to be constructed over a part of the handle, at least.

Near $\partial C, \nu(h)$ is always the identity. The second part of the construction uses this fact. Let $\dot{N}$ be the boundary of $N^{n}$ in $V^{n}$, i.e., $\dot{N}=\left(\partial N^{n}-\partial V^{n}\right)^{-}$. Since $N^{n}$ is embedded in $C$, we can assume $N^{n} \subset C$. If $N^{n}$ can be deformed in $C$ through embeddings $\xi_{t}, t \in[0,1]$, so that $U^{n}$ is always left fixed, but $\dot{N}$ is carried out to $\partial C$ (i.e., $\xi_{1}(\dot{N}) \subset \partial C$ ), then for $x$ near $\dot{N}, P \circ \nu(g(q)) \circ \xi_{1}(x)=P \circ \xi_{1}(x)$ for all $q \in I^{m}$. Using this fact, it is not too difficult to piece together an explicit formula defining $\bar{G}$. The only use of the hypotheses, condition (a) or condition (b), is in proving the existence of such a deformation $\xi_{t}$. This turns out to be simple in case (a) but case (b) is quite complicated when the index of the handle is $n$.

The details of the proof and a number of applications will be presented in a forthcoming paper.

\section{BiBLIOGRAPHY}

1. M. W. Hirsch, Immersions of manifolds, Trans. Amer. Math. Soc. 93 (1959), 242-276.

2. A. Phillips, Submersions of open manifolds, Topology 6 (1967), 171-206.

3. V. Poenaru, Regular homotopy and isotopy, mimeographed notes, Harvard University, Cambridge, Mass., 1964.

4. S. Smale, Regular curves on Riemannian manifolds, Trans. Amer. Math. Soc. 87 (1958), 492-512.

5. - Classification of immersions of spheres in Euclidean space, Ann. of Math. (2) 69 (1959), 327-344.

Cornell University 